\title{
Éditorial
}

\section{Marées noires : le risque et l'ordinaire}

La répétition de naufrages de pétroliers avec leurs conséquences sous forme de marées noires fait scandale. Il semble en effet anormal que ce secteur des transports particulièrement générateur de risques pour de larges collectivités, leur environnement et le milieu marin, ne soit pas mieux contrôlé. La disproportion apparaît même telle entre les gains liés aux risques pris par les donneurs d'ordre, les armateurs. . et les dommages éventuels, que l'analyse semble avant tout relever du simple bon sens. L'élaboration de nouvelles normes techniques (bateaux à double coque, etc.), le renforcement de la réglementation, la mise en place de nouveaux dispositifs de surveillance et de suivi du trafic à l'échelle européenne visent ainsi la suppression des «bateaux poubelles » au large de nos côtes. De façon complémentaire, l'amélioration de la gestion des situations critiques, voire de crise, semble a priori devoir suffire pour régler une grande partie des problèmes qui se posent actuellement. Et puisque, malgré tout, le pire doit être envisagé, des dispositifs visant à limiter les effets des accidents et à permettre une réparation des dommages semblent pouvoir compléter la prévention des risques. Bref, une fois éliminé l'inacceptable et installée une routine politico-administrative, il semble a priori possible d'entrer dans une véritable gestion des risques en ce qui concerne le transport maritime des matières potentiellement polluantes et, en premier lieu, du pétrole. Tout semble être affaire de raison et, si cela ne suffit pas, de sanctions.

Or, cette représentation du monde qui surgit lorsque le scandale est là est probablement loin, très loin de la réalité. En premier lieu, le risque, évident en cas d'accident, de catastrophe, n'apparaît pas aussi clairement en temps normal aux acteurs les plus immédiatement concernés. Rentabilité, continuité de services, pérennité de l'entreprise l'emportent dans un contexte défini par les contraintes des marchés et les exigences d'ordre géopolitique. Le transport maritime, comme beaucoup d'activités à risques, est ainsi un compromis entre de multiples contraintes, dangers compris. En second lieu, le risque, quelle qu'en soit la manifestation, se décline en réalité dans de multiples situations, où pannes, erreurs, incidents, quasi-accidents, accidents mineurs mais également rattrapages constants de ces divers dysfonctionnements sont le lot quotidien du transport maritime. Et cela, quels que soient le bateau ou l'équipage. En troisième lieu, l'image simplifiée du système d'acteurs qui apparaît lors d'accidents - les transporteurs, les autorités publiques, les assureurs - fait illusion sur la réalité de leurs liens. En temps normal, les acteurs a priori concernés par le transport maritime ne se positionnent pas les uns par rapport aux autres en se référant strictement aux compétences et responsabilités, mais aussi selon des modalités où les négociations et les arrangements ont toute leur importance. La sûreté du transport maritime repose davantage sur des relations faites de recommandations réciproques plutôt que d'injonctions.

Ainsi, au-delà des critiques et analyses spontanées qui se développent après chaque accident et catastrophe, de nouveaux types d'approche des problèmes posés par le transport maritime, et notamment celui mettant en jeu le pétrole, pourraient être préconisés. Il serait ainsi probablement utile de mieux savoir comment, au-delà des présentations formelles, des définitions et partages officiels de responsabilités, s'établissent effectivement les relations, les modes de coopération entre les différents types d'acteurs qui, de près ou de loin, participent au transport maritime et en définissent les modalités de « fonctionnement ». La focalisation, à bien des égards pertinente, sur les «bateaux poubelles» tend, par contraste, à orienter la réflexion sur un perfectionnement (sur le plan technologique, réglementaire, etc.) souvent hors d'atteinte et surtout sans grand rapport avec l'existant, composite, hétérogène - perfectionnement qui, au demeurant, n'est efficace que de manière transitoire. Or, c'est en partant de cette réalité, nécessairement soumise à d'assez lentes évolutions, que la gestion des risques devrait également être pensée. Une réalité faite d'accidents, certes, mais aussi de ces nombreux dysfonctionnements qui jalonnent le quotidien du transport. Resituer le risque majeur par rapport aux dangers ordinaires et aux multiples 
contraintes est probablement une des meilleures façons de ne pas l'occulter - si ce n'est de l'éviter. Enfin, peutêtre serait-il opportun de consolider ces « communautés de fait » qui se constituent à l'occasion d'une marée noire. Le pétrole déversé dans la mer, puis sur le littoral côtier met en effet en relation des mondes aux intérêts nécessairement divergents, qui se méconnaissent, mais qui ne peuvent plus complètement s'ignorer. Le capitaine grec d'un pétrolier, l'océanographe du CNRS, le pêcheur breton, l'élu d'une station balnéaire, le touriste, le citoyen « ordinaire » se trouvent ainsi ponctuellement associés par des marées noires. La question est donc de savoir comment, au sujet d'un problème qui déborde des frontières naturelles, administratives, politiques, financières, pourrait se constituer un réseau humain et organisationnel susceptible d'en épouser les divers contours.
Sans nier, donc, la nécessité de dispositifs réglementaires, de l'accroissement des contrôles et des politiques de répression visant à éviter les événements majeurs, peut-être serait-il pertinent de concevoir des actions et politiques se fondant plus sur les fonctionnements et les compromis effectifs observables dans le domaine du transport maritime. Cela ne signifierait pas accepter l'inacceptable, mais simplement ne pas se voiler la face sur la réalité actuelle et tenter de trouver dans l'ordinaire, avec ses multiples imperfections, les ressorts éventuels de la gestion de l'extraordinaire. Gestion qui passe aussi peutêtre par la structuration et la pérennité des «collectifs » produits de fait par les marées noires.

Claude Gilbert Claude.Gilbert@upmf-grenoble.fr

To access this journal online: www.edpsciences.org 